\title{
The hyperventilation syndrome: a syndrome under threat?
}

\author{
J B L Howell
}

University of Southampton, Southampton, UK

Introductory article

Double-blind placebo-controlled study of the hyperventilation provocation test and the validity of the hyperventilation syndrome

HK Hornsveld, B Garssen, MJC Fiedeldij Dop, PI van Spiegel, JCJM de Haes

Background. Hyperventilation syndrome (HVS) describes a set of somatic and psychological symptoms thought to result from episodic or chronic hyperventilation. Recognition of symptoms during the hyperventilation provocation test (HVPT) is the most widely used criterion for diagnosis of HVS. We have investigated the validity of the HVPT and of the concept of HVS. Methods. In a randomised, double-blind, crossover design, the ability of 115 patients with suspected HVS to recognise symptoms during the HVPT was compared with the ability to recognise symptoms during a placebo test (isocapnic overbreathing, with carbon dioxide levels maintained by manual titration). 30 patients who had positive results on the HVPT underwent ambulatory transcutaneous monitoring of $\mathrm{pCO}_{2}$ to ascertain whether they hyperventilated during spontaneoussymptom attacks. Findings. Of the 115 patients who underwent the HVPT and the placebo test, $85(74 \%)$ reported symptom recognition during the HVPT (positive diagnosis HVS). Of that subset, 56 were also positive on the placebo test (false-positive), and 29 were negative on the placebo test (true-positive). False-positive and true-positive patients did not differ in symptom profile or in physiological variables. During ambulatory monitoring ( 15 true-positive, 15 falsepositive) 22 attacks were registered. Transcutaneous end-tidal $\mathrm{pCO}_{2}$ decreased during only seven. The decreases were slight and apparently followed the onset of the attack, which suggests that hyperventilation is a consequence rather than a cause of the attack. There were no apparent differences between false-positive and true-positive patients. Interpretation. The HVPT is invalid as a diagnostic test for HVS. Hyperventilation seems a negligible factor in the experience of spontaneous symptoms. The term HVS should be avoided. (Lancet 1996;348:154-8)

In the introductory article by $\mathrm{H}$ ornsveld et al it is suggested that the hyperventilation provocation test (HVPT) is not diagnostically valid for the hyperventilation syndrome on the grounds that the majority $(69 \%)$ of patients with a positive HVPT were also positive following a placebo test (isocapnic hyperventilation). ${ }^{1}$ Also, during ambulatory monitoring transcutaneous end tidal carbon dioxide pressure fell in only seven of 22 spontaneous attacks, sugg suggesting that a mechanism other than hyperventilation itself is responsible for the symptoms. They also suggest that the term "hyperventilation syndrome" should be avoided.

In this review the history and origins of the hyperventilation syndrome will be presented, the proposed mechanisms of HVPT discussed, and the findings of $\mathrm{H}$ ornsveld et al put into context.

The hyperventilation syndrome

Doctors and many lay people are familiar with overbreathing associated with acute emotional reactions classically displayed by adolescent or young adult females in response to stressful situations. The patient visibly overbreathes and may show clinical signs of hypocapnia in the form of tetany. The mechanism is confirmed when the episode subsides after the patient rebreathes from a paper or plastic bag.

In contrast to this dramatic picture, there is a much commoner, more subtle form of disorder related to commen, more subtle form pe disorder related to (2) T he features are largely subjective and are not generally associated with visibly increased breathing; indeed, a disturbance of breathing is frequently not suspected and no specific diagnosis made, especially when, as is usual, the symptoms do not ring true for an organic disorder. Professor Bric when a patient's symptoms (in this case the main symptom was giddiness) do not fit into established models of disease: "T here can be few physicians so dedicated to their art that they do not experience a slight decline in spirits on learning that their patient's complaint is of giddiness. This frequently means that after exhaustive 
enquiry it will still not be entirely clear what it is that the patient feels wrong and even less so why he feels it".

I suspect the same reaction occurs with many clinicians when they attempt to assess the patient with the hyperventilation syndrome (H VS)

But why should this be so? D iagnosis involves listening to the patient's complaints and, when one or more possible causes have come to mind, directing further questioning to support or refute the possibility. Those possibilities which have not been eliminated can be pursued with further investigations. This process has been termed the hypothetico-deductive approach. ${ }^{4}$

Two major difficulties face the clinician when diagnosing a patient with HVS - firstly, thinking of the possibility and, secondly, formulating further questions to support or refute it. These difficulties stem from the lack of well recognised pathognomonic features to trigger the thought, and the absence of an adequate model of the disorder on which to base discriminating questions. Often the trigger to suspecting it will be the realisation that the clinical history is not giving to the nature of the problem - a useful time to think of HVS!

\section{Origin of the hyperventilation syndrome}

The first major account was that of K err et $\mathrm{al}^{2}$ in 1937 in which a number of case histories were recorded to illustrate the diverse clinical presentations. The account included description of a test consisting of a period of voluntary overbreathing termed "exercise in hyperventilation", in which "effort is made to induce a state of carpopedal spasm, or repetition of the symptom of the chief complaint. If no reaction is obtained within 30 minutes the procedure is discontinued". T hey appear not to have used the term "hyperventilation syndrome" themselves, but their colleagues Soley and Shock d so in their studies of the role of hypocapnia and disturbance of acid-base balance the following year. ${ }^{5} \mathrm{Sub}$ sequent reviews emphasised the relationship between symptoms and disturbance of breathing, whether the latter was merely frequent sighing or more obviously increased and sometimes disorganised breathing. The presumed mechanism of the syndrome focused on hypocapnia and for some a low arterial carbon dioxide 解 $\mathrm{H}$ owever, this was not observed in all studies, especially when the nature of the problem had not been clarified. Given this background it may come as a surprise that the name and the mechanism of the syndrome are now being called into question in this current study by H ornsveld et al. ${ }^{1}$

Their observations and conclusions need to be seen in context.

\section{Nature of the hyperventilation syndrome}

The wide range of symptoms has been described on many occasions over the past 60 years. ${ }^{27-11} \mathrm{~T}$ hey include breathlessness, dyspnoea, light-headedness, paraesthesiae, a variety of pains, especially chest pains, palpitation and sweating $M$ any of these symptoms have pal pitation and sweating. M any of these symptoms have for example, direct questioning may be needed to For example, direct questioning may be needed to elicit that attacks of breathlessness occur at rest for no apparent reason and are associated with sweating, or that the breathlessness on exertion is poorly related to the severity of the exertion, characteristically being as also unlikely to volunteer that symptoms vary within minutes or hours, and that they have "good" days when they are virtually normal. The association of breathlessness and dizziness and other somatic sensations, together with a feeling of impending loss of consciousness, 政 may be added the more general symptoms of anxiety and/or depression, and those of any organic disease that may co-exist. It is small wonder that the clinician may experience a sinking feeling in trying to fit these into a plausible diagnosis!

Physical examination may or may not reveal evidence of organic disease, because there is no reason why organic disease should leed, it may be the precipitating factor ${ }^{\prime}$ and must not be overlooked. T his emphasises that the HVS is a reaction to something psychological and/or physical which must be identified before a complete assessment can be considered to have been made.

\section{Hyperventilation provocation tests (HVPTs)}

$\mathrm{H}$ aving thought of the possibility of HVS, supported by further questions, confirmation is obtained if the symptoms are reproduced, wholly or in part, by a period of voluntary overbreathing, the rationale being that, if the spontaneous symptoms are secondary to increased ventilation, they should be reproduced by increased ventilation 12 If they ar is is difficult to attribute the symptoms to hyperventilation. There is no agreed way of conducting the voluntary overbreathing challenge. M ost accounts describe voluntary overbreathing for 1-3 minutes; my preference is for up to 20 deep breaths but $K$ err continued for as long as 30 minutes. ${ }^{2}$ In recent years some studies including those of $\mathrm{H}$ ornsveld et $\mathrm{al}^{1}$ have involved voluntary overbreathing for three minutes to produce a a manoeuvre has been termed the hyperventilation provocation test (HVPT) but the term could equally apply to other overbreathing protocols. Personally, I do not believe that such precision about duration or fall in $\mathrm{PCO}_{2}$ has been justified.

If voluntary hyperventilation reproduces the symptoms, it strongly supports the diagnosis and provides patients with an understandable explanation of their symptoms and confirmation that they are "real". M any patients are fearful that their symptoms are thought to be imaginary, contributing further to their anxiety and distress.

Some type of provocation test is vital in this "subjective" disorder because, unless clinical suspicion is supported by a convincing test, a clinician may be reluctant to exclude an organic disorder with potentially serious consequences.

MECHANISM OF HVPT

It has been widely assumed that HVPTs reproduce the patient's symptoms by inducing hypocapnia, although several years ago doubt was cast on this by G uz (personal communication). The detailed study by $\mathrm{H}$ ornsveld et $\mathrm{al}^{1}$ provides evidence that, in most subjects, the mechanism of most symptoms did not require a fall in $\mathrm{PCO}_{2}$ and, furthermore, spontaneously occurring attacks need not be associated with hypocapnia. F or these reasons they conclude that "the H VPT is invalid as a diagnostic test for HVS" and that "the term HVS has therefore become untenable". 


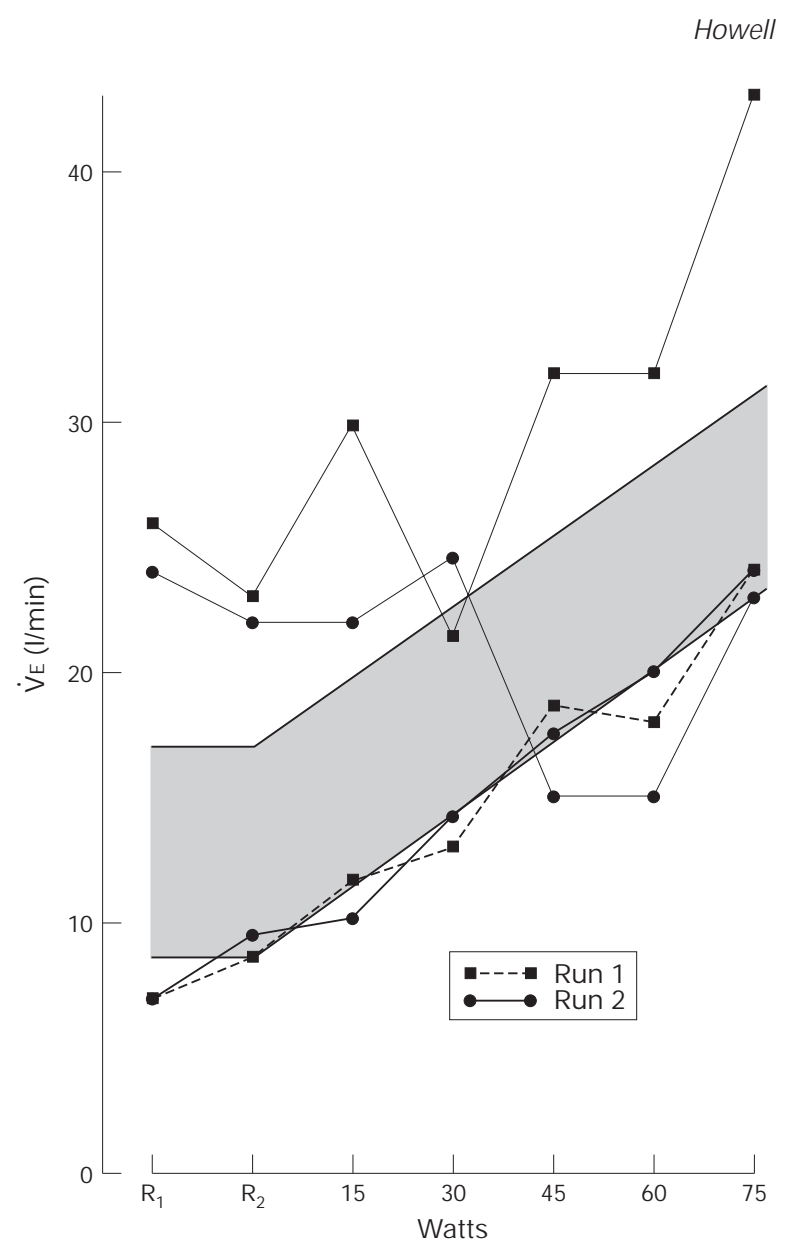

Figure 1 Ventilatory response to graded exercise on a weeks after treatment - that is, being given insight into the nature of the disorder. Two measurements were made 30 minutes apart on each occasion. The stippled area

represents the range of response seen in normal subjects. Note that before treatment the resting ventilation on one occasion was higher than at the highest level of exercise response was progressive and reproducible.

al used to define their patient group.

But if not hypocapnia, what other mechanism could generate the symptoms? Presumably it is related to the act of overbreathing itself rather than its metabolic consequences. However, before exploring sensory mechanisms associated with the act of breathing the clinical model of HVS should be considered further.

\section{A clinical model of HVS}

The extensive literature on HVS includes reports by clinicians, physiologists, psychiatrists, psychologists, and physiotherapists, most of which have been descriptive; controlled studies have been few. A recent monograph on the behavioural and psychological aspects of breathing disorders ${ }^{14}$ provides a valuable compendium of current thought while revealing that there is still insufficient information on which to base a robust model.

M y personal model of the HVS was influenced by a combined medical and psychiatric study of patients with disabling breathlessness in whom there was insufficient airflow obstruction or other form of lung or other disease to account for their symptoms. ${ }^{7}$ At that time we did not appreciate that these patients would meet the criteria for the diagnosis of the HVS. The patients had a high prevalence of depression, anxiety reactions, obsessional premorbid "perfectionist" personalities, and a smaller number had hysterical features. F urthermore, one or more of three types of experience had preceded, and may have precipitated, HVS - namely, bereavement, resentment, or disturbing uncertainty as to whether they had a serious illness. It thus became apparent that our patients with "disproportionate breathlessness" fitted the criteria for the HVS.

While most patients complain of attacks of breathlessness occurring at rest for no apparent reason, many experience breathlessness on exertion which bears little or no relation to the severity of the exercise. In a group of patients with HVS (based on clinical presentation and provocation of symptoms by 20 deep breaths) we measured the ventilatory responses to graded exercise on a bicycle ergometer before and two weeks after treatment ${ }^{15}$ which consisted solely of giving the used. Before treatment, all showed disturbance of ventilatory response to graded exercise; some hyperventilated with progressively increasing ventilation as the level of exercise increased, and others showed gross disorganisation of the breathing pattern throughout with no relationship between ventilation and leve of exercise. After treatment, at their second attendance, approximately half the patients were virtually free of symptoms and only in these had ventilatory responses to exercise returned to normal (fig 1 ). When the results, averaged for each work load, were compared in those who had and those who had not improved, the reduction in ventilation was found to be due to a reduction in the frequency of breathing rather than in tidal volume. It 政 
was clear that the abnormalities were more extensive than simple hyperventilation; the neuromuscular control of breathing was disturbed in other ways and could have contributed to their symptoms. Could this neuromuscular disturbance explain the findings of $\mathrm{H}$ ornsveld et al?

\section{Control of breathing and HVS}

The mechanisms proposed by $\mathrm{H}$ aldane and others in the early part of this century for the control of breathing are inadequate. The concept that breathing is controlled by a pontomedullary "respiratory centre" receiving afferent information from many sources, but principally from $\mathrm{CO}_{2}$ receptors on the surface of the medulla and the carotid bodies and directed at maintaining a constant $\mathrm{pH}$ in arterial blood over a wide range of metabolic activity, does not accord with many observations. Patients with primary alveolar hypoventilation (Ondine's curse) ventilate normally in response to exercise even though they cannot respond to $\mathrm{CO}_{2}$ even at high concentrations. On the other hand, some patients are unable to take a deep breath voluntarily following a stroke yet respond normally to increased concentrations stroke yet respond normally to increased concentrations
of $\mathrm{CO}_{2}$. This observation led Plum ${ }^{16}$ to conclude that, in of $\mathrm{CO}_{2}$. This observation led Plum ${ }^{16}$ to conclude that, in
addition to the "metabolic" pathway via the respiratory centre, there must also be a motor "behavioural" pathway involved in the control of breathing. This pathway presumably mediates increased ventilatory drive associated with muscular exercise when the $\mathrm{P}_{2} \mathrm{CO}_{2}$ is either (about $0.6 \mathrm{kPa}$ ) in the awake state compared with sleep and the low slope of the initial part of the $\mathrm{CO}_{2}$ response curve also implies that, when awake, the behavioural pathway is overriding $\mathrm{CO}_{2}$ chemostasis. This pathway allows control of breathing during speech, straining and other conflicting muscular manoeuvres. It is not active during non-REM sleep when the subject is reliant mainly on $\mathrm{CO}_{2}$ chemoreceptor feedback to maintain mainly on $\mathrm{CO}_{2} \mathrm{Ch}$
alveolar ventilation.

A gainst this background, we can speculate how neuromuscular activity might generate some of the symptoms of the HVS.

\section{Initiation of an attack of HVS}

For initiation of attacks a possible model might be as follows: a predisposed individual with, for example, an follows: a predisposed individual with, for example, an
obsessional personality experiences a conflict with the obsessional personality experiences a conflict with the
need for everything to be right, or one with an hysterical personality may find that the symptoms of HVS are useful in manipulating situations. T hese generatefurther emotional distress and the breathing response rapidly becomes a conditioned response

The neurophysiology of emotional disturbance is poorly understood, but emotional factors may generate nervous activity which influences that part of the sensorimotor cortex which controls the behavioural pathway described by Plum (fig 2). T his leads in turn to activation of the neuromuscular apparatus of breathing, not in the normal rhythmic way via the respiratory centre to regulate breathing to meet metabolic need but in an irregular, disorgar irregular, disorganised way unrelated to metabolic need
with tidal volume, breathing frequency, and end-exwith tidal volume, breathing frequency, and end-ex-
piratory $\mathrm{CO}_{2}$ levels varying widely. The pattern of afferent sensory information from chest wall and diaphragm receptors will be abnormal and likely to lead to bizarre sensations; muscle tension may cause pain. Of course in some patients hypocapnia does occur and is likely to contribute to symptoms, but it is not clear whether

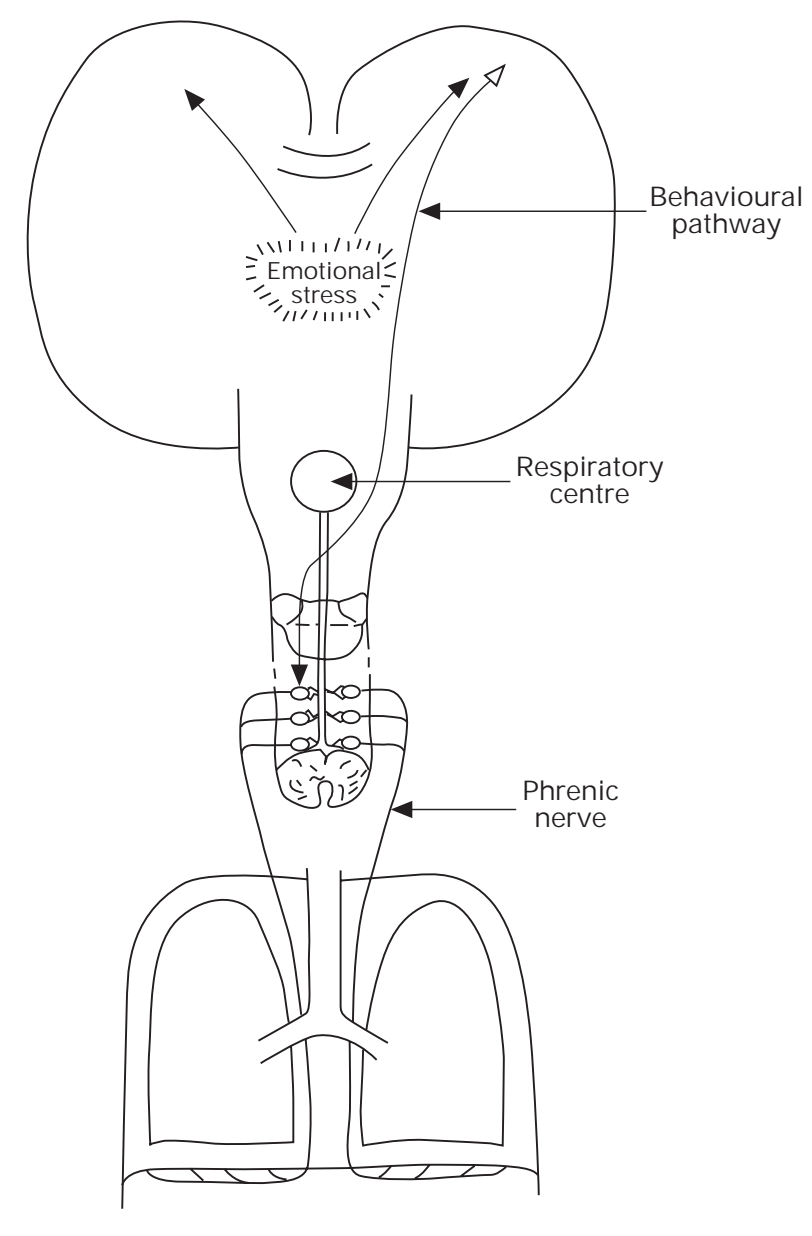

Figure 2 Schematic model of the interaction between
emotional disturbance and the behavioural pathway. symptoms such as paraesthesiae are always caused in
this way. This could be resolved by a careful analysis of symptoms generated by an HVPT, with and without the development of hypocapnia.

Of course, once a conditioned response had been established, a variety of minor stimuli might well provoke recurrent attacks of similar pattern.

\section{Sensations and muscular movement}

Current concepts of the appreciation of somatic muscular movement are relevant because breathing is under both automatic and voluntary control. When a motor command for a movement is sent from the motor cortex to spinal motor neurones, it has been proposed that a "copy" of this information - the "efference copy" - is retained within the brain where it can be compared with re-afferent proprioceptive information returning from the musculoskeletal apparatus. Any mismatch between the musculoskeletal apparatus. Any mismatch between the afferent and the efferent information is detected and
leads to corresponding sensations. This basic mechleads to corresponding sensations. This basic mech-
anism for the comparison between what is demanded of a motor act and what is achieved is believed to underlie the appreciation of sensations of weight, displacement, or resistance to movement17 in limbs. This placement, or resistance to moventin in neuromuscular model was used by Killian and C ampbel to explain the results of their extensive studies of sen-
sations of effort, force, and volume change associated with breathing under a range of experimental circumstances. They went on to propose that similar mechanisms could generate the sensations of dyspnoea: "Although dyspnea has many causes, the final common pathway resides in the proprioceptive mechanisms con- 
LEARNING POINTS

* If a patient's history is confusing, think of HVS and enquire if breathlessness is: - occurring at rest while reading, watching TV, etc

- associated with lightheadedness and paraesthesiae

- poorly related to severity of exertion

- associated with fear of dying during attacks

* Try the effect of $\mathbf{2 0}$ deep breaths. If positive, the diagnosis is almost certain.

* Avoid imposing the diagnosis. Treatment consists mainly of giving "insight" plus antidepressants, if necessary.

* Remember that HVS is a reaction to something - it may be organic disease.

trolling the respiratory muscles". ${ }^{18} \mathrm{~T}$ his model has gained wide acceptance, and it is only a small step to apply it to the generation of the symptom complex of H VS. ${ }^{19}$

\section{How would this model explain the HVPT?}

Unlike a spontaneous attack, the H VPT starts with a voluntary drive to overbreathe and one might expect the breathing patterns, and hence the sensations, to be different. But when the test reproduces the spontaneous attack it suggests that, in these subjects, voluntary overbreathing acts by triggering a conditioned response identical with the patient's spontaneous HVS.

\section{What should we call the syndrome?}

If, instead of the hyperventilation syndrome, it had been called the hypocapnic syndrome the results of $\mathrm{H}$ ornsveld et al would require us to find an alternative name. However, since there is so much evidence that the attacks are related to an underlying disturbance of breathing - frequently hyperventilation - I see no compelling reason to change the name. It is only the model pelling reason to change the name. It is only the model
whereby symptoms are generated that needs to be modiwhereby symptoms are generated that needs to be modi-
fied. In order to excite curiosity, I have sometimes used the name "behavioural breathlessness" rather than HVS $^{20}$ because the disorder is clearly a behavioural disturbance but it, too, can be misleading because many patients do not experience breathlessness as their main symptom.

\section{Conclusion}

There is little to be gained at present by changing the name of the syndrome. It is far better to be clear what we mean when we use the term HVS and to devote our efforts to understanding better the nature and mechanism of the disorder by well designed and conducted experiments such as those reported by Hornsveld et al.

1 Hornsveld H K , G arssen B, Fiedeldij D op M JC, van Spiegel PI, de H aes provocation test and the validity of the hyperventilation syndrome. Lancet 1996;348:154-8.
$K$ err WW, Dalton JW, G liebe PA. Some physical phenomena associated
with anxiety states and their relation to hyperventilation. Ann Intern M ed 1937; 111 1961-92.
a thews WB. Practical neurology. Oxford: Blackwell Scientific, 1963: $3 M$ athews WB. Practical neurology. Oxford: Blackwell Scientific, 163 .

4 Campbell EJM. The diagnosing mind. L ancet 1987;i: 849-51. 5 Soley M, Shock $\mathrm{N}$. The aetiology of effort syndrome. Am J M ed SC
$1938 ; 196: 840-51$. $G$ ardner WN, M eah M M, Bass C. Controlled study of respiratory
responses during prolonged measurement in patients with chronic hyperventilation. Lancet 1986;ii: $826-30$.

7 Burns BH, Howell JBL. Disproportionately severe breathlessness in . Ames F. The hyperventilation syndrome. J M ent Sci 1955; 101-466-525.
Rice RL. Symptom patterns of the hyperventilation syndrome. A I J
M ed 1950;8:691-700.

10 Brashear RE. H yperventilation syndrome. L ung 1983; 161:257-73. 1975; 19.375-83.

12 Lewis RA, Howell JBL. D efinition of hyperventilation syndrome. Bull $H$ ornsveld $H$ G arssen $B$ Fienteldi D

, van Spiegel P. Symptom reporting during voluntary hyperventilation and mental load: im-
plications for diagnosing hyperventilation syndrome. J Psychosom Res 4 Timmons $B H$,

breathing disorders $R$, eds. Behavioural and psychological approaches to 5 Patel S, A A disoded T. B B ehavioural breathlessness and the control of vent-

Plum F. N eurological integration of behavioural and metabolic control Symposium. London: Churchill, 1970:159-81.

In: American Physiological Society, 1981:1415-47.

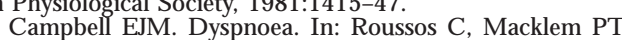
Howell IBL. Breathlessness. In: Brewis RAL, Corrin B, G eddes DM,
Gibson GJ, eds. Respiratory medicine. 2nd edn. London: WB Saunders, H owell JBL. Behavioural breathlessness. Thorax 1990;45:287-92. 